\title{
Measuring small absorptions by exploiting photothermal self-phase modulation
}

\author{
Nico Lastzka, Jessica Steinlechner, Sebastian Steinlechner, and Roman Schnabel ${ }^{\star}$ \\ Institut für Gravitationsphysik, Leibniz Universität Hannover and Max-Planck-Institut für Gravitationsphysik \\ (Albert-Einstein-Institut), Callinstrasse 38, 30167 Hannover, Germany \\ ${ }^{\star}$ Corresponding author: roman.schnabel@aei.mpg.de
}

Received 25 May 2010; accepted 9 August 2010; posted 1 September 2010 (Doc. ID 128994); published 27 September 2010

\begin{abstract}
We present a method for the measurement of small optical absorption coefficients. The method exploits the deformation of cavity Airy peaks that occur if the cavity contains an absorbing material with a nonzero thermorefractive coefficient $\mathrm{d} n / \mathrm{d} T$ or a nonzero expansion coefficient $a_{\mathrm{th}}$. Light absorption leads to a local temperature change and to an intensity-dependent phase shift, i.e., to a photothermal self-phase modulation. The absorption coefficient is derived from a comparison of time-resolved measurements with a numerical time-domain simulation applying a Markov-chain Monte Carlo algorithm. We apply our method to the absorption coefficient of lithium niobate doped with $7 \mathrm{~mol}$. \% magnesium oxide and derive a value of $\alpha_{\mathrm{LN}}=(5.9 \pm 0.9) \times 10^{-4} / \mathrm{cm}$. Our method should also apply to materials with much lower absorption coefficients. Based on our modeling, we estimate that, with cavity finesse values of the order of $10^{4}$, absorption coefficients of as low as $10^{-8} / \mathrm{cm}$ can be measured. (๑) 2010 Optical Society of America OCIS codes: $\quad 120.0120,120.5060,120.6810$.
\end{abstract}

\section{Introduction}

Materials with low optical absorption coefficients are essential for high-precision laser-interferometric measurements. Absorptions in mirror substrates of as low as $10^{-6} / \mathrm{cm}$ already limit gravitational wave detectors because absorption leads to heating and a thermal deformation of the mirrors [1] and also to photothermal noise [2]. Future gravitational wave detectors will use cryogenically cooled mirrors [3] to reduce thermally excited motions of mirror surfaces. Then, low optical absorptions will become even more crucial. Consequently, the measurement of small absorption coefficients in the regime below $10^{-6} / \mathrm{cm}$ is important to find appropriate mirror materials and to enable the reliable design of future gravitational wave detectors, such as the Einstein Telescope [4, $\underline{5}]$.

In the past, several methods have been developed that are able to measure absorption coefficients of the order of $10^{-6} / \mathrm{cm}$. All these methods are based on indirect measurement schemes. They do not di-

0003-6935/10/285391-08 $\$ 15.00 / 0$

(C) 2010 Optical Society of America rectly sense the power loss of a transmitted beam but utilize the temperature increase that arises due to the absorption. In calorimetric approaches, the temperature increase is directly measured [6]. Other approaches exploit light beam deflection or beam shape deformation due to local heating $[7,8]$.

In this paper we present another indirect measurement scheme to determine small absorptions. The material under investigation is put inside an optical cavity whose length is linearly scanned over a cavity Airy peak. Approaching cavity resonance, the temperature along the cavity mode increases and the optical path length for a cavity round trip changes. The thermally induced optical path length change is a photothermal self-phase modulation resulting in a deformed shape of the Airy peak. The actual phase change depends on the light intensity, which is similar to what is observed for the optical Kerr effect. Importantly, the Airy peak deformation depends on the scan direction, i.e., whether the cavity is shortened or lengthened. The hysteresis in the timeresolved measurements provides information of the absorption coefficient, if relevant material parameters are known and included in a numerical 
time-domain simulation. A positive side effect of our method is the power buildup inside the cavity, which compensates the need for laser sources with higher powers when approaching the regime of extremely low absorption.

\section{Theory and Method}

In this section we describe the time-domain simulation that is used to analyze the measurement data and to deduce the absorption from it. Our approach is based on work by Hello and Vinet $[9,10]$ in which they describe the heating of an absorbing material due to a Gaussian laser beam. In our case, a sample of the absorbing material with polished (plane) surfaces is placed inside a (high) finesse cavity. One may choose the light's angle of incidence to be the Brewster angle to avoid reflection losses. A schematic is shown in Fig. 1. When the cavity round-trip phase $\phi_{\text {cav }}$ is linearly increased (or reduced) by $\delta(t)$ and scanned over a cavity resonance, absorption leads to a dynamic temperature profile inside the material and inside the cavity mirror surfaces. The result is a (photothermal) self-phase modulation and a deformation of the cavity Airy peak.

Our time-domain model iteratively calculates the intracavity intensity after each round trip. The time $t$ is discretized, becoming an integer multiple of the
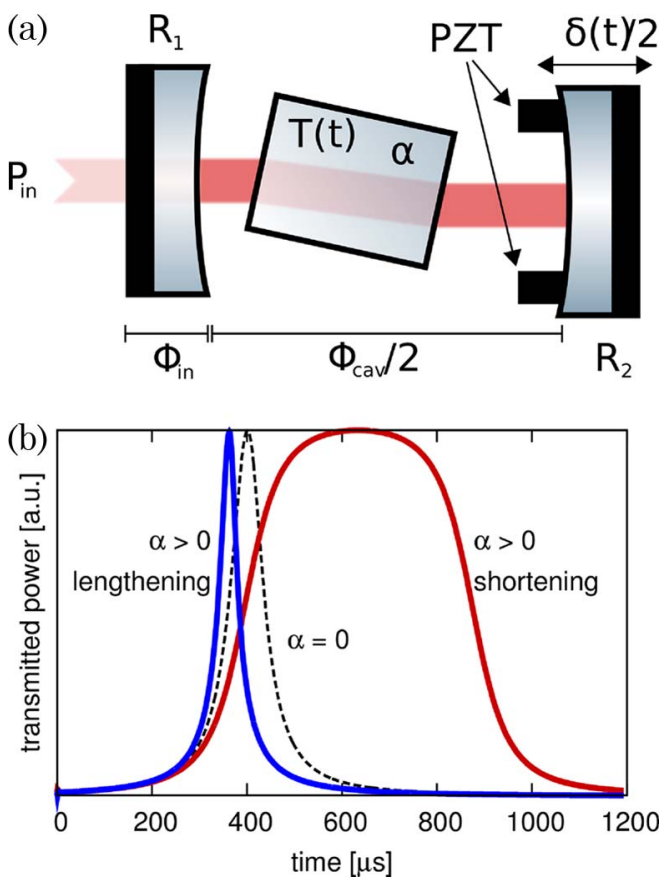

Fig. 1. (Color online) Scheme of the measurement method. (a) When changing the cavity round-trip phase by $\delta(t)$, absorption of intracavity power leads to a temperature change $T(t)$ and an additional phase for the intracavity field $\phi_{\text {cav }} . R_{1}$ and $R_{2}$ are the mirror power reflectivities; $\phi_{\text {in }}$ is the input phase. By using the Brewster angle, surface reflections can be avoided. (b) Airy peaks showing hysteresis due to the photothermal self-phase modulation. The dashed curve shows the peak without any absorption $(\alpha=0)$, whereas the blue (narrow) and red (broad) peaks show the Airy peak for the same absorption coefficient $\alpha>0$, but for lengthening and shortening the cavity, respectively. round-trip time, yielding $t=t_{n}=n / \Delta f_{\mathrm{FSR}}$, where $\Delta f_{\mathrm{FSR}}$ is the cavity free spectral range. The intracavity field $a_{n}:=a\left(t_{n}\right)$ after $n$ round trips reads

$$
a_{n}=\mathrm{i} \sqrt{1-r_{1}^{2}} a_{\mathrm{in}} \mathrm{e}^{\mathrm{i} \phi_{\mathrm{in}}\left(t_{n}\right)}+r_{1} \tilde{r}_{2} \mathrm{e}^{\mathrm{i} \phi_{n}\left(t_{n}\right)} a_{n-1}
$$

Here, $r_{1}$ is the amplitude reflectivity of the first mirror, whereas $\tilde{r}_{2}$ is the effective amplitude reflectivity of the second mirror, which includes all round-trip losses. The amplitude of the incident power $P_{\text {in }}$ is given by $a_{\text {in }}=2 \sqrt{P_{\text {in }} /\left(\epsilon_{0} c \pi w^{2}\right)}$, where $w$ is the waist radius of the beam at the entrance of the cavity, $\epsilon_{0}$ is the dielectric constant, and $c$ is the speed of light. Note that the calculation of the temperature gradient inside the substrates assumes a constant waist size $w$. In an actual experiment, the Rayleigh range of the field, therefore, must be much greater than the substrate dimension multiplied by its refractive index. The cavity input field gains the phase $\phi_{\text {in }}$ that is due to the temperature gradient inside the incoupling mirror emerging from its coating absorption. The phase $\phi_{n}\left(t_{n}\right)$ after $n$ round trips can be written as

$$
\phi_{n}=\delta\left(t_{n}\right)+\phi_{\mathrm{spm}}\left(t_{n}, \alpha\right),
$$

where $\delta\left(t_{n}\right)$ is the phase due to the external cavity detuning and $\phi_{\mathrm{spm}}\left(t_{n}, \alpha\right)$ is due to the photothermal (internal) self-phase modulation, which depends on the absorption $\alpha$. The external detuning for the round-trip number $n$ is determined from

$$
\delta\left(t_{n}\right)=\delta_{0}+n 2 \pi N_{\mathrm{FSR}} \frac{\omega_{s}}{\Delta f_{\mathrm{FSR}}} .
$$

Here, $N_{\text {FSR }}$ is the number of free spectral ranges that were scanned with frequency $\omega_{s}$. The velocity $v_{m}$ of the scanning mirror is, therefore, given by

$$
v_{m}=2 \lambda \omega_{s} \cdot N_{\mathrm{FSR}} .
$$

The temperature distribution $T_{n}$ for round-trip $n$ is calculated by using the recurrence relations [Eqs. (15) in [10]\}. These equations determine the radial and longitudinal temperature gradient at any time, including thermal conductivity. The starting point is the external temperature $T_{0}$. The detuning $\phi_{\mathrm{spm}}$ induced by the photothermal self-phase-modulation is then given by Eqs. (33) and (35) of [9]. Note that $\phi_{\mathrm{spm}}$ includes the effects of a nonzero thermo-optic coefficient $\mathrm{d} n / \mathrm{d} T$ and a nonzero expansion coefficient $a_{\mathrm{th}}$. As a starting point for the numerical simulation, we use the steady-state solution for the start detuning $\delta_{0}$ :

$$
a_{0}=\mathrm{i} \sqrt{1-r_{1}^{2}} \frac{a_{\mathrm{in}}}{1-r_{1} \tilde{r}_{2} \mathrm{e}^{\mathrm{i} \delta_{0}}} .
$$

Assuming a perfect mode matching of the input field $a_{\text {in }}$ to the cavity mode, the reflected and transmitted fields are given by 


$$
\begin{gathered}
a_{\text {refl }}\left(t_{n}\right)=\mathrm{i} \sqrt{1-r_{1}^{2}} a_{n}(\alpha)+r_{1} a_{\text {in }} \mathrm{e}^{\mathrm{i} \phi_{\text {in }}}, \\
a_{\text {trans }}\left(t_{n}\right)=\mathrm{i} \sqrt{1-\tilde{r}_{2}^{2}} a_{n}(\alpha) .
\end{gathered}
$$

Equation (5) can now be used to calculate the timeresolved shape of an Airy peak. By varying $\alpha$, the result can be fitted to the measurement performed in reflection or transmission of the cavity. Figure 1(b) shows an example, i.e., simulated Airy peaks obtained from a cavity containing some absorbing material with $\mathrm{d} n / \mathrm{d} T>0$. The resonance peaks get broader when the cavity is shortened because the positive thermorefractive coefficient counteracts the external change of the cavity length. Accordingly, the resonance peaks get narrower when the cavity length is increased. In particular, the hysteresis can be used to precisely determine the absorption of the material. For comparison we also show the normal Airy peak without self-phase modulation (dashed curve).

\section{Measurements and Data Analysis}

To characterize the feasibility of our method, we performed a series of absorption measurements on a 7 mol. \% MgO-doped $\mathrm{LiNbO}_{3}$ (LN) crystal. A single measurement set involves a characterization of the piezoelectric element that is used to change the cavity length, and, altogether, four time-resolved photoelectric detections. A fast photodiode records the Airy peaks in reflection of the cavity when the latter is (a) lengthened or (b) shortened, using (1) a low laser power without any thermal Airy peak deformation or (2) a laser power at which a thermal deformation is clearly visible. The low-power setting is used to quantify the two reflectivities $r_{1}^{2}$ and $\tilde{r}_{2}^{2}$. The highpower setting is used to quantify the absorption coefficient $\alpha_{\mathrm{LN}}$. All three quantities and their error bars are deduced from a single measurement set and a numerical time-domain simulation applying a Markovchain Monte Carlo (MCMC) algorithm. Records (a1) and (b1) are identical, thereby, confirming that the laser power was chosen to be low enough that thermal effects do not yet come into play. Note that (a1) and (b1) will not necessarily have the shape of the central Airy peak (dashed curve) shown in Fig. 1(b) but may show a ringing effect due to the cavity loading or decay time [11]. This effect is also precisely modeled in our simulation.

\section{A. Experimental Setup}

In our research group, we routinely use cavities containing MgO-doped LN crystals for second harmonic generation (SHG) and squeezed light generation at a wavelength of $1064 \mathrm{~nm}$ [12-14]. The optical absorption of these nonlinear crystals is a limiting factor in achieving high conversion efficiencies and high squeezing factors. Accurate absorption coefficients are therefore required to optimize the nonlinear cavity design. Unfortunately, manufacturers' data typically are rather inaccurate and a standard value of
$\alpha_{\mathrm{LN}} \lesssim 10^{-3} / \mathrm{cm}$ at $1064 \mathrm{~nm}$ is quoted in most cases. In this work we used one of our SHG cavities to measure the absorption coefficient of LN and test our new absorption measurement technique.

Figure 2 shows the experimental setup. An incoupling mirror and the curved side of the planoconvex $\mathrm{LiNbO}_{3}$ crystal form a single-ended standing wave cavity for laser light at $1064 \mathrm{~nm}$. The cavity mirrors have power reflectivities of $r_{1}^{2}=R_{1} \approx 90 \%$ and $r_{2}^{2}=R_{2}>99.8 \%$. A small air gap separates the in-coupling mirror from the antireflection coated plane crystal surface. Table 1 contains detailed geometric parameters of this resonator and the laser beam as well as the material parameters of the LN crystal.

Up to $1.5 \mathrm{~W}$ of single-mode radiation at $1064 \mathrm{~nm}$ was mode matched into the cavity with a modematching efficiency of greater than $95 \%$. To prevent the generation of second harmonic radiation, both the input field polarization and the crystal temperature were detuned from their usual operation point. A piezoelectric transducer (PZT) moved the incoupling mirror to allow for a scan of the cavity length. The photodiode measured the temporal behavior of the reflected laser power. We ensured that the photodiode was fast enough, i.e., had a high bandwidth, so that it did not influence the shape of the recorded Airy peaks.

Figure 3 shows an example of Airy peaks with visible thermal effects as measured in reflection of the cavity. The blue curve forms for a lengthening resonator, the red curve for a shortening resonator. No parameter other than the scan direction was changed. The two curves would be identical without selfphase modulation and no hysteresis effect would occur without absorption. The solid curves in Fig. 3 represent our simulation fitted to the experimental data. The narrow curves show a discrepancy in the left wings, the broad curves show a discrepancy in the right wings. The two deviations come from the imperfect mode matching to the cavity and the excitation of a higher-order cavity mode.

Apart from taking a simple full measurement set, i.e., lengthened and shortened resonator at two different laser powers, we performed measurements at three different laser powers and three different

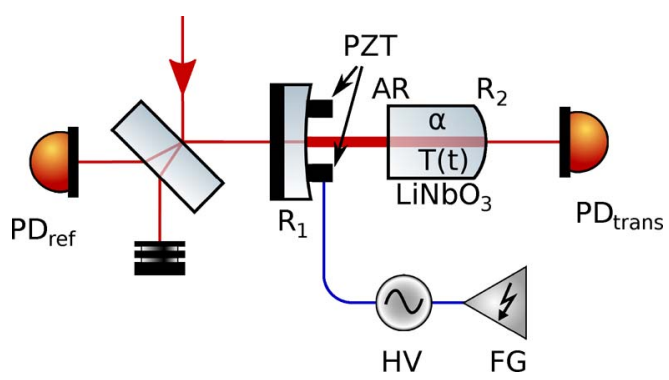

Fig. 2. (Color online) Experimental setup: the resonator is formed by the in-coupling mirror with reflectivity $R_{1} \approx 90 \%$ and the crystal's highly reflecting coating with a reflectivity of $R_{2}>99.8 \%$. The resonator length is scanned with a frequency from a function generator (FG) which is fed through a high voltage amplifier (HV). 
Table 1. Material and Geometric Parameters of the $\mathrm{LiNbO}_{3}$ and Si Samples and Cavity Geometric Parameters Used for the Simulations

\begin{tabular}{|c|c|c|}
\hline Material Parameters & $\mathrm{LiNbO}_{3}$ & $\mathrm{Si}$ \\
\hline Thermal refr. coeff. $\mathrm{d} n / \mathrm{d} T$ & $38.5 \cdot 10^{-6} / \overline{\mathrm{K}}[17]$ & $176.0 \cdot 10^{-6} / \mathrm{K}[\underline{16}]$ \\
\hline Density $\rho$ & $4635 \mathrm{~kg} / \mathrm{m}^{3}[\underline{20]}$ & $2330 \mathrm{~kg} / \mathrm{m}^{3}[\underline{18}]$ \\
\hline Thermal expansion $a_{\mathrm{th}}$ & $14.8 \cdot 10^{-6} / \mathrm{K}[\underline{18}]$ & $2.53 \cdot 10^{-6} / \mathrm{K}[\underline{19}]$ \\
\hline Thermal conductivity $k_{\mathrm{th}}$ & $4.19 \mathrm{~W} /(\mathrm{mK})[21]$ & $1.56 \mathrm{~W} /(\mathrm{mK})[\underline{22}]$ \\
\hline Material emissivity $\epsilon$ & $1.0^{a}$ & $1.0^{a}$ \\
\hline \multicolumn{3}{|l|}{ Cavity Geometric Parameters } \\
\hline Air gap $s$ & $24 \mathrm{~mm}$ & $0 \mathrm{~mm}$ \\
\hline Beam waist $\omega_{0}$ & $24 \mu \mathrm{m}$ & $160 \mu \mathrm{m}$ \\
\hline Crystal length $L$ & $6.5 \mathrm{~mm}$ & $65.0 \mathrm{~mm}$ \\
\hline Crystal radius $R$ & $2 \mathrm{~mm}$ & $50.0 \mathrm{~mm}$ \\
\hline
\end{tabular}

${ }^{a} 0.0<€ \leq 1.0$ are the boundaries for the thermal emissivity. For our systems, the value of this parameter is not relevant since $R \gg \omega_{0}$.

scan frequencies. While not strictly necessary for an absorption measurement, these measurements demonstrate the consistency of our result; see Subsection 3.B.

\section{B. Measurement Analysis}

For the analysis of the measured peaks, the PZT had to be calibrated because of its own hysteresis and nonlinearity. This calibration was done at low laser powers where no thermal effect occurred. We measured the width of the Airy peaks at different positions of the PZT's scanning range by slightly shifting the laser frequency. A third-degree polynomial well described the peak width depending on peak position. Together with a scan showing a full free spectral range, we used this polynomial to linearize the PZT movement. We performed the calibration for both scan directions and for each scan velocity that we used. Measurements were performed at three different scan velocities, namely, $2 \cdot 1064 \mathrm{~nm} / 5 \mathrm{~ms}, 2 \cdot 1064 \mathrm{~nm} / 2.5 \mathrm{~ms}$, and $2 \cdot 1064 \mathrm{~nm} / 0.285 \mathrm{~ms}$. For each scan velocity, we measured Airy peaks at three different input powers: 100 $\mathrm{mW}, 750 \mathrm{~mW}$, and $1.5 \mathrm{~W}$.

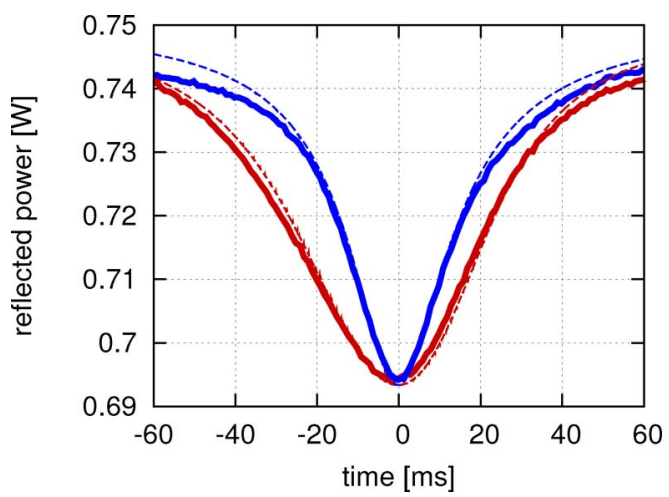

Fig. 3. (Color online) Example of measured (solid curves) and simulated (dashed curves) Airy peaks with visible thermal effect. Without absorption, all curves would be identical. The curves were measured in reflection; no parameter other than the scan direction was changed. The red (broad) curve forms for a shortening resonator, the blue one (narrow) for a lengthening resonator.
Table 1 gives a complete list of the parameters that enter our simulation. We used values from literature for the material parameters [15-22]; for the geometric parameters, we chose values based on our best knowledge of the cavity design. The mirror reflectivities $R_{1}$ and $\tilde{R}_{2}$ define the cavity resonance width and power buildup. Here, $\tilde{R}_{2}$ is an effective reflectivity, which includes absorption and scattering losses. Only this value, rather than the pure reflectivity $R_{2}$, is accessible when light enters the cavity through mirror $R_{1}$. As resonance width and power buildup have a strong impact on the heating of the substrate, we do not use the reflectivity values as given by the coating manufacturer. Instead, we treat $R_{1}$ and $\tilde{R}_{2}$, as well as the absorption $\alpha_{\mathrm{LN}}$, as free parameters of our simulation.

For low input powers and fast scan velocities, the resulting temperature change inside the substrate is small and no deformation of the peaks is visible. Such time series are optimally suited to determine $R_{1}$ and $\tilde{R}_{2}$. Toward higher input powers and lower scan velocities, the peaks begin to show a hysteresis. For all our measurements performed with different laser powers, the hysteresis values could be explained completely by the self-phase modulation, i.e., our simulation provided a very good description of the measurement. From this we conclude that no spatial deformation of the cavity mode occurred.

We performed a quantitative analysis by calculating the variance between simulated and measured data. Starting from an initial set of parameters, we ran a Metropolis-Hastings MCMC [23] algorithm, which minimized the variance. The data chains generated can be converted into histograms for the free simulation parameters. The histograms for reflectivities $R_{1}$ and $\tilde{R}_{2}$ and for the absorption $\alpha_{\mathrm{LN}}$ as derived from a single measurement setting are shown in Fig. 4. As the histograms closely resemble Gaussian distributions, we give the mean value and standard deviation of all nine measurements in Table 2.

The mean value of the results for the in-coupling mirror was found to be $R_{1}=(89.43 \pm 0.75) \%$, which 

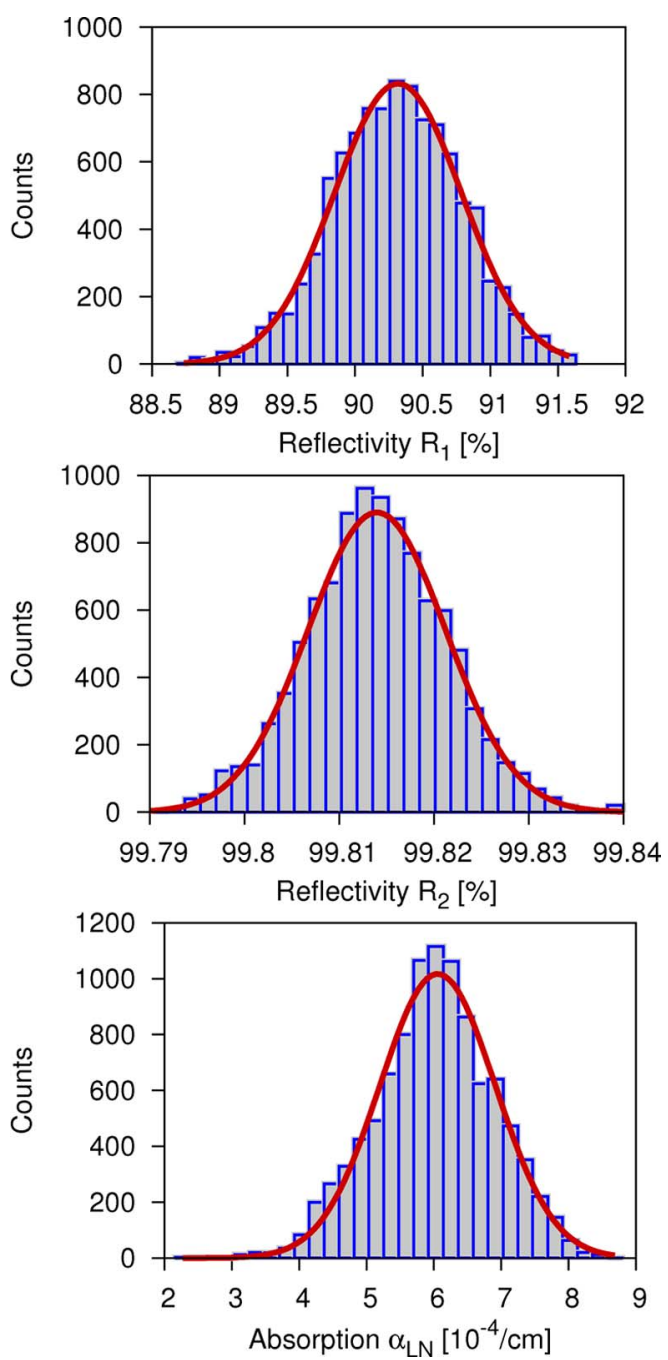

Fig. 4. (Color online) The Metropolis-Hastings MCMC algorithm draws samples from the simulation parameter space, creating a chain of individual realizations that result in the parameter distributions. Here we show the histograms of a chain obtained from a single measurement set at a laser input power of $0.75 \mathrm{~W}$ and a scan-velocity of $v=2 \cdot 1064 \mathrm{~nm} / 2.5 \mathrm{~ms}$. $R_{1}$ (top) and $\tilde{R}_{2}$ (middle) are required to characterize the cavity. The bottom figure shows the result for $\alpha_{\mathrm{LN}}$. The bars represent histograms of the MCMC run. The curves are Gaussian fits to the histograms.

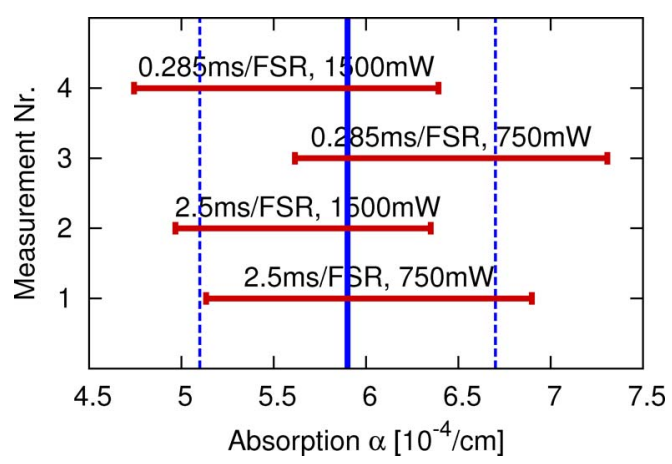

Fig. 5. (Color online) Four independent measurement values of the absorption coefficient $\alpha_{\mathrm{LN}}$ and their statistical standard deviations. The blue vertical line shows the mean value of the four measurements, which is $\alpha_{\mathrm{LN}}=5.9 \times 10^{-4} / \mathrm{cm}$. The dashed vertical blue lines mark the averaged standard deviation of $\Delta \alpha_{\mathrm{LN}}=0.8 \times 10^{-4} / \mathrm{cm}$.

is in good agreement with the manufacturer's specification for this coating $[(90 \pm 1) \%]$. The effective value for the high-reflective coating of the crystal was determined to be $\tilde{R}_{2}=(99.79 \pm 0.01) \%$, which is also in accordance with the specifications. The measurement with $100 \mathrm{~mW}$ input power at a scan velocity of $v=2$. $1064 \mathrm{~nm} / 5 \mathrm{~ms}$ was the boundary where a small thermal effect was visible. However, no accurate absorption coefficient could be deduced due to rather large error bars. Four measurements showed a significant thermal effect and were used to derive four independent values for the absorption coefficient of LN. All four values for $\alpha_{\mathrm{LN}}$ have mutually overlapping error bars. Figure 5 gives a graphical overview of the results for $\alpha_{\mathrm{LN}}$ for all measurements. The mean value of the four results is $\alpha_{\mathrm{LN}}=5.9 \times 10^{-4} / \mathrm{cm}$. As the error bar, we quote the standard deviation (an averaged value) of the single measurement set, which typically was $\pm 0.8 \times 10^{-4} / \mathrm{cm}$. This number includes the influence from errors in the reflectivities $R_{1}$ and $\tilde{R}_{2}$, as shown in Fig. $\underline{4}$.

\section{Error Propagation}

We considered the influence of possible errors in the input parameters on the resulting value for $\alpha_{\mathrm{LN}}$ (from a single measurement). For this investigation we individually changed the values of the simulation

Table 2. Results for $R_{1}, \tilde{R}_{2}$, and $\alpha_{\mathrm{LN}}{ }^{a}$

\begin{tabular}{|c|c|c|c|c|c|c|c|}
\hline \multirow[b]{2}{*}{$f$ in $\mathrm{ms} / \Delta f_{\mathrm{FSR}}$} & \multirow[b]{2}{*}{$P$ in $\mathrm{W}$} & \multicolumn{2}{|c|}{$R_{1}$} & \multicolumn{2}{|c|}{$\tilde{R}_{2}$} & \multicolumn{2}{|c|}{$\alpha_{\mathrm{LN}}$ in $10^{-4} / \mathrm{cm}$} \\
\hline & & $\bar{R}_{1}$ & $\Delta R_{1} \cdot 10^{3}$ & $\overline{\tilde{R}}_{2}$ & $\Delta \tilde{R}_{2} \cdot 10^{5}$ & $\bar{\alpha}_{\mathrm{LN}}$ & $\Delta \alpha_{\mathrm{LN}}$ \\
\hline 0.285 & 0.1 & 0.89668 & 6.46 & 0.99812 & 8.58 & - & - \\
\hline 0.285 & 0.75 & 0.89585 & 5.37 & 0.99793 & 8.3 & - & - \\
\hline 0.285 & 1.5 & 0.88532 & 3.62 & 0.99786 & 5.12 & - & - \\
\hline 2.5 & 0.1 & 0.8957 & 19.2 & 0.99802 & 26.6 & - & - \\
\hline 2.5 & 0.75 & 0.90316 & 4.68 & 0.99814 & 1.27 & 6.016 & 0.8828 \\
\hline 2.5 & 1.5 & 0.88438 & 7.74 & 0.9978 & 12.2 & 5.5685 & 0.692 \\
\hline 5 & 0.1 & 0.90153 & 5.26 & 0.99804 & 7.89 & $(10.247)$ & $(4.39)$ \\
\hline 5 & 0.75 & 0.89401 & 7.41 & 0.99797 & 11.5 & 6.4605 & 0.846 \\
\hline 5 & 1.5 & 0.892 & 11.8 & 0.99746 & 24.1 & 5.5672 & 0.824 \\
\hline
\end{tabular}

\footnotetext{
${ }^{a}$ Mean values and standard deviation of the parameters are given.
} 
input parameters and recalculated $R_{1}, \tilde{R}_{2}$, and $\alpha_{\mathrm{LN}}$ for each case.

Our investigation showed that the parameters can be grouped into two categories. The first category contains parameters that have a very weak influence on the absorption coefficient in our case. For our system, heat radiation described by the material emissivity $0.0<\epsilon \leq 1.0$ is not relevant at all, because the substrate is heated only within the beam radius, far away from the substrate's surface. Also, the absorption coefficient of the substrate coatings $\left(\alpha_{\text {coating }}\right)$ can be neglected, as it is much smaller than the substrate absorption $\alpha_{\mathrm{LN}}$ and the coating thickness is negligible compared to the substrate dimension. A few percent change of the values for the index of refraction $n$, the intracavity air gap $s$, the substrate radius $R$, and the beam waist $\omega_{0}$ also has a negligible effect on the absorption coefficient $\alpha_{\mathrm{LN}}$. The second category contains the remaining parameters of our model. These parameters and their respective influence on $\alpha_{\mathrm{LN}}$ for a $4 \%$ change in the parameter value are the input laser power $P(3.6 \%)$, the substrate length $L(3.6 \%)$, the thermal conductivity $k_{\mathrm{th}}(1.6 \%)$, the thermal refractive coefficient $\mathrm{d} n / \mathrm{d} T$ (3.6\%), the thermal expansion $a_{\mathrm{th}}(1.4 \%)$, the density $\rho$, and the heat capacity $c$ $(2.8 \%)$. Note that, in the simulation, $\rho$ and $c$ always appear as a product, and the influence of their error bars is identical.

Assuming that our measured parameters, as well as the material parameters from literature, are precise to within $4 \%$ and statistically independent from each other, we conclude that the error of $\pm 0.8 \times$ $10^{-4} / \mathrm{cm}( \pm 13.6 \%)$ coming directly out of the MCMC simulation dominates the error on our final result. The total error sums up to $\pm 0.9 \times 10^{-4} / \mathrm{cm}( \pm 15.7 \%)$.

\section{Sensitivity of the Method}

To make a prediction of the sensitivity of our method, we consider the absorption measurement of crystalline silicon at a wavelength of $1550 \mathrm{~nm}$. This value has not been measured before, but data at shorter wavelengths $[24,25]$ suggest an absorption coefficient smaller than $10^{-8} / \mathrm{cm}$ in the case of pure silicon [26]. Our simulation is based on a $6.5 \mathrm{~cm}$ long silicon sample inside a cavity of finesse 20,000 pumped with $1 \mathrm{~W}$ of input laser power. The reflectivities of the two cavity mirrors are assumed to be identical. Figure $\underline{6}$ a shows the Airy peaks as detected in the reflected light for both cavity scan directions with an absorption of $10^{-8} / \mathrm{cm}$. The scan velocity used in that simulation was $v_{\mathrm{m}}=2 \cdot 1550 \mathrm{~nm} / \mathrm{s}$. The curves are normalized to the input power of $1 \mathrm{~W}$. Both curves show oscillations and values above unity that arise from the cavity loading and decay time [11]. Figure $6(\mathrm{~b})$ shows the difference of the two scan directions normalized to the Airy peak without absorption. We find a significant hysteresis curve that reaches up to $12 \%$ of the input power. Our simulation neglects the influence of the absorption in the dielectric coatings. In practice, the absorption inside the cavity mirror coatings has to be insignificant, as in our experiment, or it has to be mea-
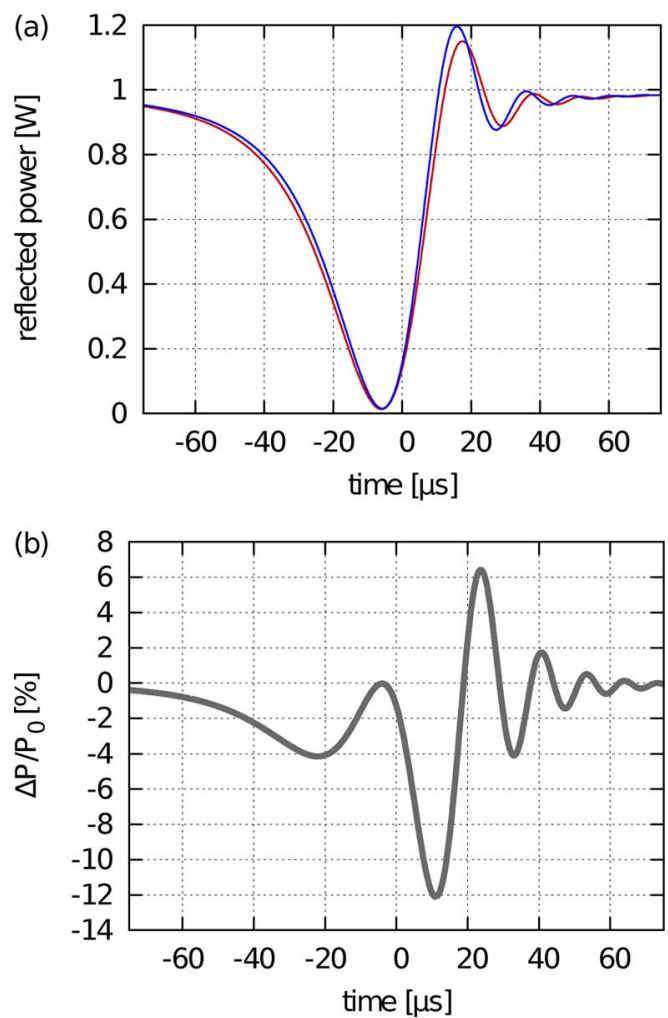

Fig. 6. (Color online) Simulated hysteresis effect for the Airy peaks in reflection from a monolithic silicon cavity of finesse 20,000 . The curves are normalized to the input power of $1 \mathrm{~W}$ at $1550 \mathrm{~nm}$. The scan velocity of the cavity length is $2 \cdot 1550 \mathrm{~nm} / \mathrm{s}$ and the absorption was assumed to be $10^{-8} / \mathrm{cm}$. Other parameters can be found in Table 1. (a) Airy peaks for lengthening (blue/upper) and shortening (red/lower) the cavity. (b) The difference of the two scan directions $\Delta P$ normalized to the incident laser power of $1 \mathrm{~W}$.

sured independently when the sample is removed from the cavity. Antireflection coatings or highreflection coatings on the sample itself can also be taken into account when two different sample lengths are studied. Generally, the photothermal self-phase modulation from the coating absorption must not dominate the overall photothermal effect inside the cavity. New low-loss coating materials, such as diamond [27] or monolithic, nanostructured surfaces [28], might be used.

\section{Conclusion}

In this paper we introduce a low-absorption measurement method based on the optical phase change inside the material when absorption leads to local heating. The effect is understood as a cavity-assisted photothermal self-phase modulation of light. The material under investigation is put inside an optical cavity whose length is linearly scanned over a cavity Airy peak. The intracavity laser power buildup leads to heating and a deformation of the Airy peak, which provides information about the absorption. We used our method to determine the absorption coefficient $\alpha_{\mathrm{LN}}$ of a LN crystal. Our result of $\alpha_{\mathrm{LN}}=(5.9 \pm$ $0.9) \times 10^{-4} / \mathrm{cm}$ is in accordance with the typically referred upper bound of $10^{-3} / \mathrm{cm}$, as available on 
manufacturer websites. Measurements with different laser powers and different scanning speeds could all be well described without considering other effects, in particular a spatial mode distortion. We conclude that no such mode conversion occurred in our experiments. However, this might be possible at even higher laser powers or smaller waist sizes. Suitable cavity finesse and scanning speed values can be deduced from the two scenarios presented. Generally speaking, the finesse should provide a significant power buildup. Mirror reflectivities should be chosen such that the cavity is always overcoupled within an estimated range of possible round-trip losses. However, the precise finesse value is not crucial, since the scanning speed can be optimized online. Using this parameter, the Airy peak deformation and its hysteresis can be adjusted to be significant but not too high to meet the modeling assumptions made. We theoretically applied our method to a material with an absorption coefficient of $\alpha=10^{-8} / \mathrm{cm}$. We conclude that such low absorptions should be measurable when a sample of a few centimeters in length is put into a cavity with a finesse of the order of $10^{4}$. Our time-resolved MCMC simulation is based on a variety of material parameters. The coupling of parameter errors into the error of the absorption coefficient is linear or less.

We thank Harald Lück for many valuable remarks on the manuscript. This research was supported by the Centre for Quantum Engineering and SpaceTime Research (QUEST). We also acknowledge funding from the International Max Planck Research School (IMPRS) on Gravitational Wave Astronomy.

\section{References}

1. W. Winkler, K. Danzmann, A. Rüdiger, and R. Schilling, "Heating by optical absorption and the performance of interferometric gravitational-wave detectors," Phys. Rev. A 44, 7022-7036 (1991).

2. V. B. Braginsky, M. L. Gorodetsky, and S. P. Vyatchanin, "Thermodynamical fluctuations and photo-thermal shot noise in gravitational wave antennae," Phys. Lett. A 264, 1-10 (1999).

3. K. Arai, R. Takahashi, D. Tatsumi, K. Izumi, Y. Wakabayashi, H. Ishizaki, M. Fukushima, T. Yamazaki, M.-K. Fujimoto, A. Takamori, K. Tsubono, R. DeSalvo, A. Bertolini, S. Márka, V. Sannibale, the TAMACollaboration, T.Uchiyama, O. Miyakawa, S. Miyoki, K. Agatsuma, T. Saito, M. Ohashi, K. Kuroda, I. Nakatani, S. Telada, K. Yamamoto, T. Tomaru, T. Suzuki, T. Haruyama, N. Sato, A. Yamamoto, T. Shintomi, the CLIO Collaboration, and the LCGTCollaboration, "Status of Japanese gravitational wave detectors," Class. Quantum Grav. 26, 204020 (2009).

4. M. Punturo, M. Abernathy, F. Acernese, B. Allen, N. Andersson, K. Arun, F. Barone, B. Barr, M. Barsuglia, M. Beker, N. Beveridge, S. Birindelli, S. Bose, L. Bosi, S. Braccini, C. Bradaschia, T. Bulik, , G. Cella, E. Chassande Mottin, S. Chelkowski, A. Chincarini, J. Clark, E. Coccia, C. Colacino, J. Colas, A. Cumming, L. Cunningham, E. Cuoco, S. Danilishin, K. Danzmann, G. De Luca, R. De Salvo, T. Dent, R. Derosa, L. Di Fiore, A. Di Virgilio, M. Doets, V. Fafone, P. Falferi, R. Flaminio, J. Franc, F. Frasconi, A. Freise, P. Fulda, J. Gair, G. Gemme, A. Gennai, A. Giazotto, K. Glampedakis, M. Granata, H. Grote, G. Guidi, G. Hammond, M. Hannam, J. Harms, D. Heinert, M.
Hendry, I. Heng, E. Hennes, S. Hild, J. Hough, S. Husa, S. Huttner, G. Jones, F. Khalili, K. Kokeyama, K. Kokkotas, B. Krishnan, M. Lorenzini, H. Lück, E. Majorana, I. Mandel, V. Mandic, I. Martin, C. Michel, Y. Minenkov, N. Morgado, S. Mosca, B. Mours, H. Müller-Ebhardt, P. Murray, R. Nawrodt, J. Nelson, R. Oshaughnessy, C. DOtt, C. Palomba, A. Paoli, G. Parguez, A. Pasqualetti, R. Passaquieti, D. Passuello, L. Pinard, R. Poggiani, P. Popolizio, M. Prato, P. Puppo, D. Rabeling, P. Rapagnani, J. Read, T. Regimbau, H. Rehbein, S. Reid, L. Rezzolla, F. Ricci, F. Richard, A. Rocchi, S. Rowan, A. Rüdiger, B. Sassolas, B. Sathyaprakash, R. Schnabel, C. Schwarz, P. Seidel, A. Sintes, K. Somiya, F. Speirits, K. Strain, S. Strigin, P. Sutton, S. Tarabrin, J. van den Brand, C. van Leewen, M. van Veggel, C. van den Broeck, A. Vecchio, J. Veitch, F. Vetrano, A. Vicere, S. Vyatchanin, B. Willke, G. Woan, P. Wolfango, and K. Yamamoto, "The third generation of gravitational wave observatories and their science reach," Class. Quantum Grav. 27, 084007 (2010).

5. http://www.et-gw.eu

6. U. Willamowski, T. Gross, D. Ristau, and H. Welling, "Calorimetric measurement of optical absorption at $532 \mathrm{~nm}$ and $1064 \mathrm{~nm}$ according to ISO/FDIS 11551," Proc. SPIE 2870, 483-494 (1996).

7. V. Loriette and C. Boccara, "Absorption of low-loss optical materials measured at $1064 \mathrm{~nm}$ by a position-modulated collinear photothermal detection technique," Appl. Opt. 42, 649-656 (2003).

8. S. Hild, H. Lück, W. Winkler, K. Strain, H. Grote, J. Smith, M. Malec, M. Hewitson, B. Willke, J. Hough, and K. Danzmann, "Measurement of a low-absorption sample of $\mathrm{OH}$-reduced fused silica," Appl. Opt. 45, 7269-7272 (2006).

9. P. Hello and J. Vinet, "Analytical models of thermal aberrations in massive mirrors heated by high power laser beams," J. Phys. (France) 51, 1267-1282 (1990).

10. P. Hello and J. Vinet, "Numerical model of transient thermal effects in high power optical resonators," J. Phys. (France) 3, 717-732 (1993).

11. L. Matone, M. Barsuglia, F. Bondu, F. Cavalier, H. Heitmann, and N. Man, "Finesse and mirror speed measurement for a suspended Fabry Perot cavity using the ringing effect," Phys. Lett. A 271, 314-318 (2000).

12. S. Chelkowski, H. Vahlbruch, K. Danzmann, and R. Schnabel, "Coherent control of broadband vacuum squeezing," Phys. Rev. A 75, 043814 (2007).

13. H. Vahlbruch, M. Mehmet, S. Chelkowski, B. Hage, A. Franzen, N. Lastzka, S. Gossler, K. Danzmann, and R. Schnabel, "Observation of squeezed light with $10 \mathrm{~dB}$ quantum-noise reduction," Phys. Rev. Lett. 100, 033602 (2008).

14. H. Vahlbruch, "Squeezed light for gravitational wave astronomy," Ph.D. dissertation (University of Hannover, 2008).

15. D. E. Zelmon, D. L. Small, and D. Jundt, "Infrared corrected Sellmeier coefficients for congruently grown lithium niobate and 5 mol. \% magnesium oxide-doped lithium niobate," J. Opt. Soc. Am. B 14, 3319-3322 (1997).

16. B. J. Frey, D. B. Leviton, and T. J. Madison, "Temperaturedependent refractive index of silicon and germanium," Proc. SPIE 6273, 62732J (2006).

17. D. H. Jundt, M. M. Fejer, and R. L. Byer, "Optical properties of lithium-rich lithium niobate fabricated by vapor transport equilibration," IEEE J. Quantum Electron. 26, 135-138 (1990).

18. M. J. Weber, Handbook of Optical Materials (CRC, 2003).

19. R. Hull, ed., Properties of Crystalline Silicon (INSPEC, 1999).

20. I. W. Kim, B. C. Park, B. M. Jin, A. S. Bhalla, and J. W. Kim, "Characteristics of $\mathrm{MgO}$-doped $\mathrm{LiNbO}_{3}$ crystals," Mater. Lett. 24, 157-160 (1995).

21. K. K. Wong, ed., Properties of Lithium Niobate (INSPEC, 2002). 
22. C. J. Glassbrenner and G. A. Slack, "Thermal conductivity of silicon and germanium from $3{ }^{\circ} \mathrm{K}$ to the melting point," Phys. Rev. 134, A1058-A1069 (1964).

23. P. Gregory, Bayesian Logical Data Analysis for the Physical Sciences (Cambridge U. Press, 2005).

24. M. Green and M. Keevers, "Optical properties of intrinsic silicon at $300 \mathrm{~K}$," Prog. Photovolt. Res. Appl. 3, 189192 (1995).

25. M. Keevers and M. Green, "Absorption edge of silicon from solar cell spectral response measurements," Appl. Phys. Lett. 66, 174-176 (1995).

26. R. Schnabel,M. Britzger, F. Brückner, O. Burmeister, K. Danzmann, J. Dück, T. Eberle, D. Friedrich, H. Lück, M.
Mehmet, R. Nawrodt, S. Steinlechner, and B. Willke, "Building blocks for future detectors: silicon test masses and $1550 \mathrm{~nm}$ laser light," arxiv:0912.3164 (2009)

27. B. V. Spitsyn, L. L. Bouilov, and B. V. Derjaguin, "Vapor growth of diamond on diamond and other surfaces," J. Cryst. Growth 52, 219-226 (1981).

28. F. Brückner, D. Friedrich, T. Clausnitzer, M. Britzger, O. Burmeister, K. Danzmann, E.-B. Kley, A. Tünnermann, and R. Schnabel, "Realization of a monolithic high-reflectivity cavity mirror from a single silicon crystal," Phys. Rev. Lett. 104, 163903 (2010). 